\title{
Erratum: Obstructive Hydrocephalus Due to Unruptured Brain Arteriovenous Malformation: Demonstrating Transcranial Color Duplex Confirmation of Cerebral Venous Hemodynamic Alterations and Color Duplex Ultrasound Confirmation of Shunt Patency
}

\author{
Kathryn J. Busch ${ }^{1}$, Hosen Kiat ${ }^{2}$, Alberto Avolio ${ }^{3}$, Mark Butlin ${ }^{4}$, Andrew Davidson ${ }^{5}$ \\ 1. Miscellaneous, Faculty of Medicine and Health Sciences, Macquarie University, Sydney, AUS 2. \\ Cardiology, Faculty of Medicine and Health Sciences, Macquarie University, Sydney, AUS 3. \\ Cardiovascular Research, Department of Biomedical Sciences, Faculty of Medicine and Health Sciences, \\ Macquarie University, Sydney, AUS 4. Cardiology, Department of Biomedical Sciences, Faculty of \\ Medicine and Health Sciences, Macquarie University, Sydney, AUS 5. Neurosurgery, Faculty of Medicine \\ and Health Sciences, Macquarie University, Sydney, AUS
}

$\square$ Corresponding author: Kathryn J. Busch, kathrynjbusch@gmail.com

Corresponding author: Kathryn J. Busch

\begin{abstract}
1. Miscellaneous, Faculty of Medicine and Health Sciences, Macquarie University, Sydney, AUS 2. Cardiology, Faculty of Medicine and Health Sciences, Macquarie University, Sydney, AUS 3. Faculty of Medicine, University of New South Wales, Sydney, AUS 4. Cardiovascular Research, Department of Biomedical Sciences, Faculty of Medicine and Health Sciences, Macquarie University, Sydney, AUS 5. Cardiology, Department of Biomedical Sciences, Faculty of Medicine and Health Sciences, Macquarie University, Sydney, AUS 6. Neurosurgery, Faculty of Medicine and Health Sciences, Macquarie University, Sydney, AUS
\end{abstract}

How to cite this erratum

Busch K J, Kiat H, Avolio A, et al. (January 06, 2020) Correction: Obstructive Hydrocephalus Due to Unruptured Brain Arteriovenous Malformation: Demonstrating Transcranial Color Duplex Confirmation of Cerebral Venous Hemodynamic Alterations and Color Duplex Ultrasound Confirmation of Shunt Patency. Cureus 12(1): c26. doi:10.7759/cureus.c26

\section{Correction Notice}

The following errors have been corrected (all located in the three paragraphs between Figures 6 and 7 in the Case Presentation section):

First paragraph: "(Figure 4C)" reference removed and "(Figure 6A, 6B)" reference added.

Second paragraph: "Pressure support ventilation" replaced with "peak systemic velocity", "volume" replaced with "velocity", and "(Figure 7)" reference added.

Third paragraph: "VM" replaced with "mean velocity" and "(Figure 7)" reference removed. 